\title{
Is Cost-Related Non-Adherence to Medical Care Unique to the USA?
}

\section{James X Zhang*}

Department of Medicine, The University of Chicago, 5841 S. Maryland Ave., MC 5000, Chicago, IL, USA

\section{Editorial}

Cost-Related Non-Adherence (CRN) to medical care is a serious and persistent challenge in the USA. For example, twenty-six percent of the elderly does not take medication as prescribed due to a cost barrier [1]. This is not surprising, as many elderly patients depend on a small amount of fixed income from social security and struggle on a day-to-day basis to make difficult choices between meeting the basic needs of daily life and filling prescriptions. However, cost-related non-adherence to medication is prevalent across adult age strata. For example, 35 million (19\%) Americans aged between 19 and 64 had not filled a prescription because of cost in 2014 [2].

Although much of the literature focuses on the CRN rate in medication use, CRN behaviors are much broader than not filling prescriptions. For example, in 2014, 23\% of adults had not gone to the doctor when they were sick because of cost; $19 \%$ skipped a recommended test, treatment, or follow-up visit because of cost; and $13 \%$ had not got needed care from a specialist because of cost. Combining all these four categories of CRN, including not filling prescriptions, $36 \%$ of American adults engaged in one or more CRN behaviors in 2014 [2].

Thus far, the majority of literature in CRN has focused on the populations in the USA. However, there is burgeoning body of research on cross-sectional comparison in CRN behaviors among developed countries. For example, in a study of adults aged 55 and older and living in the community in 11 developed countries, the authors found that following the lead of the USA with $16.8 \%$ in CRN to medication, Canada had the second highest national prevalence of CRN $(8.3 \%)$, followed by Australia (6.8\%) [3]. While the prevalence of CRN among all older adults were much lower in France, Norway, Sweden, Switzerland and the UK, the same study showed that CRN rates vary by income, indicating a combination of effects on access to medication by income and additional insurance coverage due to low income (i.e., additional public insurance coverage associated with low income may have offset the effect of low income).

Taking CRN broadly including any cost-related access problem to medical care among adults aged 18 or older, the prevalence of CRN rates is $33 \%$ in the USA. According to another recent study, followed by Switzerland (22\%), New Zealand (18\%), France (17\%), Canada (16\%), Australia (14\%), Norway (10\%), Netherland (8\%), Sweden (8\%), UK (7\%), and Germany (7\%) [4]. The same study also shows significant variation in CRN rates by income in each of those countries.

While much more needs to be ascertained in order to explain the variation in CRN rates, it is clear that insurance coverage plays a pivotal role in enabling patients' access to the needed care, often offsetting the effect of poverty. For example, the lower income population in UK had lower CRN rate in medication, largely due to the additional pubic insurance coverage to lower their out-of-pocket burden of medication. Beyond health insurance, because CRN is a patient behavior based on each individual's decision-making process, many socio-economic factors other than health can play a role, including education, race, and last but not least, gender [5]. The efforts to reduce CRN should be focused on the tailored-approach on specific factors that influence the variations in CRN rates. In this regard, there is much more to be learned in advancing the knowledge of CRN behaviors across the international settings.

\section{References}

1. Wilson IB, Schoen C, Neuman P, Strollo MK, Chang H, et al. (2007) Physicianpatient communication about prescription medication nonadherence: A 50-state study of America's Seniors. J Gen Intern Med 22: 6-12.

2. Collins SR, Rasmussen PW, Doty MM, Beutel S (2015) Rise in health care coverage and affordability since health reform took effect findings from the commonwealth fund biennial health insurance survey, 2014, The Commonwealth fund issue brief.

3. Morgan SG, Lee A (2017) Cost-related non-adherence to prescribed medicines among older adults: A cross sectional analysis of a survey in 11 developed countries. Health Serv Res e014287.

4. Osborn R, Squires D, Doty MM, Sarnak DO, Schneider EC (2016) In new survey of eleven countries, US adults still struggle with access to and affordability of health care. Health Aff 35: 2327-2336.

5. Zhang JX, Crowe JM, Meltzer DO (2017) Differential rates in cost-related nonadherence to medical care by gender in the USA adult population. J Med Econ 20: 752-759.
*Corresponding author: James X Zhang, Department of Medicine, The University of Chicago, 5841 S. Maryland Ave., MC 5000, Chicago, IL, USA, Tel: 1-7738341631; E-mail: xzhang1@medicine.bsd.uchicago.edu

Received August 22, 2017; Accepted August 23, 2017; Published August 31 2017

Citation: Zhang JX (2017) Is Cost-Related Non-Adherence to Medical Care Unique to the USA? J Gerontol Geriatr Res 6: e146. doi:10.4172/2167-7182.1000e146

Copyright: (๑) 2017 Zhang JX. This is an open-access article distributed under the terms of the Creative Commons Attribution License, which permits unrestricted use, distribution, and reproduction in any medium, provided the original author and source are credited. 